http://dx.doi.org/10.1590/0104-1428.1582

\title{
Usinabilidade de Materiais Compósitos Poliméricos para Aplicações Automotivas
}

\author{
Juan Carlos Horta Gutiérrez, Juan Carlos Campos Rubio, Paulo Eustáquio de Faria \\ Universidade Federal de Minas Gerais - UFMG, Brasil

\section{Departamento de Engenharia Mecânica, Universidade de Aveiro, Portugal}

\begin{abstract}
Resumo: A cada dia o avanço tecnológico nos leva a encruzilhadas como a construção de veículos mais leves, com menor consumo de combustível e mais seguros. Neste sentido, materiais compósitos poliméricos são materiais usados atualmente em diferentes aplicações nas quais a leveza combinada com um alto módulo de elasticidade são características importantes. Poliamidas reforçadas com fibras de vidro se apresentam como materiais de grande futuro em aplicações automotivas. Desta forma, torna-se importante o estudo do comportamento deste tipo de material quando submetidos a processos de usinagem. Dentre os processos de usinagem o processo de furação é um dos mais utilizados nos componentes de material compósito. Neste trabalho será analisada a influência do avanço, da velocidade de corte e da geometria da ferramenta na força de avanço, pressão específica de corte e desvios dimensionais do furo realizado. Os testes foram realizados em poliamida sem reforço (PA6) e reforçada com 30\% fibra de vidro (PA66-GF30) utilizando brocas de carbonetos sinterizados (K20) com ângulos de ponta diferente. Os resultados apontam claramente para a melhor usinabilidade do material reforçado em comparação ao sem reforço.
\end{abstract}

Palavras-chave: Compósitos, polimeros reforçados, usinabilidade, furação, danos.

\section{Machining Behavior of Polymer Composites Materials for Automotive Applications}

\begin{abstract}
The continuous technological advance has led to the challenge of building lighter and safer vehicles, but with lower fuel consumption. Within this scenario, polymeric composites are materials currently used in different applications, where lightweight combined with high modulus of elasticity are important features. Polyamide reinforced with glass fiber face a promising future for automotive applications, thus becoming important to study the behavior of this type of material when subjected to machining processes. Among the machining processes, drilling is one of the most widely applied to composite materials. In this work the influence of feed rate, cutting speed and tool geometry on thrust force, specific cutting pressure and hole dimensional deviations is investigated. The tests were performed on unreinforced polyamide (PA6) and on polyamide reinforced with $30 \mathrm{wt} \%$ glass fiber (PA66-GF30) using tungsten carbide drills (K20) with different tip angles. The results clearly indicate the superior machinability of the reinforced material, compared with the unreinforced polyamide.
\end{abstract}

Keywords: Composites, reinforced polymers, machinability, drilling, damage.

\section{Introdução}

Os polímeros são macromoléculas de elevada massa molecular, resultante de reações químicas de polimerização e formadas por unidades menores denominadas monômeros. Polímeros podem ser de origem natural, derivados de substâncias obtidas de plantas, animais ou minerais, ou de origem sintética, criados a partir de produtos petroquímicos ou silicones. Polímeros podem ser classificados em dois grupos: termoplásticos e termorrígidos. Termoplásticos se caracterizam por permitir ser fundido na presença de calor e endurecido por resfriamento diversas vezes, alguns deles podem igualmente dissolverem com o uso de solventes, o que facilita sua reciclagem. Por causa de sua reação irreversível de polimerização, os polímeros termorrígidos apresentam características de elevada rigidez, dureza e freqüentemente fragilidade, entretanto o aquecimento provoca a decomposição do material, dificultando sua reciclagem ${ }^{[1]}$
Apesar dos materiais poliméricos reforçados (compósitos de matriz poliméricos) serem empregados em aplicações de engenharia desde a primeira década do século XX, foi a partir da década de 40 que a sua utilização pela indústria voltada para o setor militar passou a apresentar crescimento acentuado, seguida mais tarde por outros diversos segmentos da indústria de bens de consumo, a partir do momento em que o custo desses materiais deixou de ser proibitivo. Aplicações típicas desses materiais incluem componentes aeronáuticos, automotivos e náuticos, estruturas marítimas, tubulações, componentes eletrônicos e ainda equipamentos militares e esportivos, particularmente em situações onde elevada relação entre resistência mecânica (ou rigidez) e massa é desejada.

Os materiais compósitos, em geral, se apresentam como uma combinação de duas ou mais fases insolúveis e distintas quimicamente, e cujas propriedades e

Autor para correspondência: Juan Carlos Campos Rubio, Departamento de Engenharia Mecânica, Universidade Federal de Minas Gerais - UFMG, Av. Antônio Carlos, 6627, Campus Pampulha, CEP 31270-901, Belo Horizonte, MG, Brasil, e-mail: juan@ufmg.br 
desempenho são superiores às desses constituintes quando atuando isoladamente. Através da adição de reforços na forma de fibras de vidro, carbono (grafite), kevlar, entre outros, se obtém uma melhoria apreciável de algumas das principais propriedades dos materiais poliméricos, tais como resistência mecânica, rigidez e resistência à fluência.

O material compósito possuirá propriedades que dependem mutuamente das fibras, material de reforço, e da matriz. Cada propriedade, porém, é influenciada de maneira diferente. A resistência à tração, por exemplo, é, basicamente, função da fibra de reforço, enquanto a capacidade de absorção de energia em um impacto depende mais da matriz. O compósito, entretanto, é a junção das duas partes, e pode-se demonstrar isso pelo fato de que, quando algumas fibras se rompem, a deformação elástica ou plástica da matriz produz forças de cisalhamento que gradualmente fazem os fragmentos de fibra exercer resistência novamente. Desse modo, percebe-se que, na ausência da matriz, as fibras ao redor teriam de suportar um aumento maior de tensão o que tornaria o material mais frágil ${ }^{[2,3]}$.

Quanto às fibras, não apenas o tipo de fibra influencia nas propriedades de um material compósito, a geometria das fibras de reforço também o faz. Um exemplo disso é o fato de que fibras longas são preferíveis para a construção de compósitos. Isso ocorre porque a carga é transferida da matriz para as fibras através de forças de cisalhamento que ocorrem na superfície, ou seja, quando maior for a superfície em relação à seção transversal da fibra, melhor essa transferência de carga. Se a relação raio-comprimento não é suficiente o compósito irá falhar a uma carga menor do que a que ele teoricamente poderia suportar, pois as fibras se soltarão da matriz sem se romperem e o compósito de desintegrará. Existe um segundo motivo para que fibras longas sejam preferíveis, sua orientação pode ser controlada de modo que o compósito pode ser projetado de modo a antecipar as cargas que ele irá enfrentar ${ }^{[4]}$.

Mais um processo em destaque devido a sua rapidez é a moldagem por injeção de resina ou Resin Transfer Molding (RTM). Utilizando esse processo, primeiramente tecidos de fibra de reforço são colocados no molde da peça a ser construída. Após essa etapa, resina é injetada no molde sob pressão para posteriormente a peça ser curada. Depois disso, ela já pode ser desmoldada e utilizada em sua aplicação ${ }^{[5]}$.

Outro processo produtivo de grande crescimento é a utilização de placas de compósitos de 1 a $2 \mathrm{~mm}$ de espessura que depois podem ser conformadas para obtenção de formas complexas através da superposição de placas e moldagem através da utilização de pressão. Um exemplo da utilização desse processo é a fabricação de elementos especiais de absorção de energia que ficam localizados atrás do pára-choque. Esses elementos são fabricados com base nas lâminas de compósitos que são prensadas em formato de chapéu e que depois são unidas duas a duas pelas laterais. Experimentos indicam que para elementos no formato de perfil de duplo chapéu, os compósitos de matrizes termoplásticas são os que mais absorvem energia ${ }^{[6]}$. Esse melhor comportamento em batidas já é explorado na Fórmula 1 há algum tempo, o que resultou em um menor número de acidentes fatais nesses últimos anos.
Em seu trabalho, Ghassemieh ${ }^{[5]}$ afirma que os materiais usados na indústria automotiva precisam cumprir estritos critérios antes de serem aprovados. Atender a normas vigentes, não só de resistência, mas também relativas ao impacto ambiental, segurança e exigências de conforto de usuários. Diversas vezes fatores avaliados são conflitantes e um projeto adequado pode ser possível por meio de soluções otimizadas e/ou hibridas que contemplem aspectos positivos de mais de um material.

$\mathrm{Na}$ indústria automotiva, a substituição dos materiais tradicionais por compósitos poliméricos de alta tecnologia está levando a uma série de vantagens como a diminuição no peso total do carro, o aumento na segurança dos passageiros e diminuição de manutenção, tudo isso aliado a uma diminuição nos custos e nas etapas do processo produtivo ${ }^{[7]}$. Isso ocorre devido ao grande módulo de elasticidade e grande capacidade de absorção de energia em conjunto com uma baixa densidade ${ }^{[8]}$.

Além de todos os benefícios supracitados, os compósitos estão relacionados com uma economia verde. Estima-se que cada $10 \%$ de redução em massa de um veículo resulta na economia de 6 a $8 \%$ de combustível. Essa economia é muito mais fácil de alcançar e demanda muito menos dinheiro do que pesquisas em formas alternativas de motores e combustíveis. Outra vantagem da utilização desse tipo de material é que a redução de peso pode levar uma menor necessidade de potência nos veículos, o que levaria a uma redução nos motores, nas baterias, entre outros sistemas, gerando, assim, um gasto energético menor ainda. Devido a esses motivos, é previsto um grande crescimento para o mercado de compósitos ${ }^{[2]}$.

A Figura 1 mostra alguns exemplos de componentes automotivos fabricados em plásticos reforçados com fibras. Dentre os exemplos atuais da aplicação desta tecnologia pode-se destacar a fabricação de chassis, suportes de fixação e célula habitáculo para passageiros como no Porsche Carrera GT e Mercedes-Benz SLR McLaren feita de compósito polimérico com fibra de carbono (Figura 1a,b). Outros automóveis esportivos de alto desempenho como o Bugatti - Veyron utilizam as características de relação peso/rigidez dos compósitos poliméricos para aumentar o desempenho, a utilização de spoilers e aerofólios permitem a diminuição da turbulência e o aumento da força contra o solo durante a aceleração, e na frenagem ao inclinar o aerofólio em $60^{\circ}$ torna-se um freio aerodinâmico de grande eficiência (Figura 1c). Igualmente, polímeros reforçados com fibra de vidro vêm sendo utilizados na fabricação de componentes como: suportes de fixação, sistemas de amortecimentos de impactos, pára-choques e sistemas de admissão de gases. Matrizes poliméricas reforçadas com fibra de carbono começam a ter sua utilização disseminada para a fabricação de pára-lamas, grades, capôs e portas de abertura vertical, como em automóveis Ford Mustang e BMW (Figura 1d-f) ${ }^{[9]}$.

Material compósito reforçado com fibra de carbono desenvolvido pela Lamborghini em conjunto com a Boeing, é utilizado na construção do chassi, quadro, rodas e assentos do Lamborghini Aventador LP700.

A Figura $1 \mathrm{~g}$ mostra um exemplo de assentos esportivos fabricados em Aramida (Kevlar), os quais permitem uma 


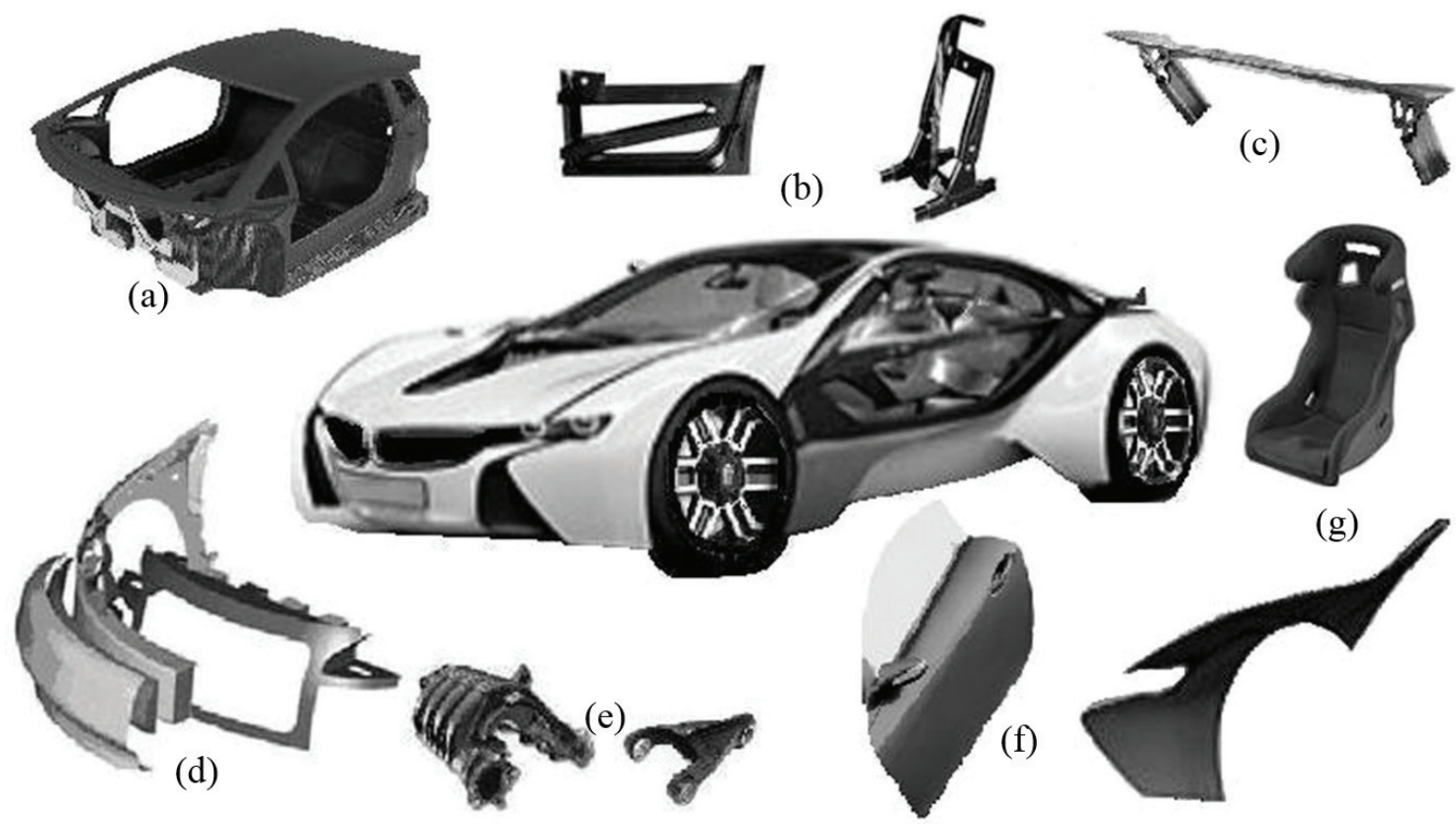

Figura 1. Exemplo de aplicações de PRF em componentes automotivos.

maior resistência ao fogo (chama). Quadros de PVC (Cloreto de Polivinila) reforçados com fibra de carbono ou poliamidas (PA) reforçada com fibra de vidro tem uso em componentes estruturais, por exemplo, motocicletas de Trial.

Motocicletas de alto desempenho para corridas de competição aparecem como uma das proeminentes áreas da indústria automotiva para uso de materiais compósitos poliméricos. Um exemplo é a Buell 1190 RS Carbon Edition com apenas $175 \mathrm{~kg}$ e $185 \mathrm{cv}$ de potência. Como o nome sugere, esta motocicleta apresenta uma versão especial com muitas peças em fibra de carbono, incluindo a carroceria, sistema de exaustão, radiador e suportes de carenagem.

Da mesma forma, existe a possibilidade de utilizar carroçaria de fibra de vidro para atender os requisitos da Associação Americana de Motociclistas - AMA Superbike Pro, onde a carroceria de fibra de carbono e os discos de freio de fibra de carbono não são permitidos nas corridas, devido a seu desempenho superior ${ }^{[10]}$.

Os compósitos poliméricos, portanto, possuem um grande potencial para construções de baixo peso e elevada resistência e, além disso, possui uma variada gama de propriedades isotrópicas e anisotrópicas dependentes dos tipos de fibra e matrizes a serem utilizadas e da geometria da utilização. Prognósticos sobre o futuro do mercado dos compósitos poliméricos são positivos e apontam para uma maior diversificação de seu uso, prevendo um crescimento na área nanocompósitos como, por exemplo, pela adição de nanotubos de carbono em matrizes poliméricas.

\section{Poliamidas Reforçadas e sua Usinagem}

Entre os polímeros industrialmente utilizados, as poliamidas (PA) ou náilons, como são mais conhecidos, vem sendo muito utilizados em soluções de engenharia, devido à versatilidade de compor inúmeras formulações com excelentes balanços de propriedades.

Estas resinas semicristalinas, da família dos termoplásticos, são resultado da combinação de uma diamina e um diácido, como no caso do náilon 6.6 (PA 66), ou derivada de um aminoácido de lactama, como no náilon 6 (PA 6). Os números de átomos de carbono presentes no diácido e diamina, ou no aminoácido, determinam o tipo de PA, sendo a PA 6.6 (polímero de adipato de hexametilenodiamina, ou sal N, com seis carbonos no diácido original - ácido adípico - e seis carbonos na diamina) e a PA 6 (com seis carbonos de lactama) as mais tradicionais e com maior aplicação no mercado mundial. Extensa, a família ainda agrega as especialidades PA 4.6, PA 10, PA 11, PA 12, entre outras, diferenciadas pelo melhor desempenho de umas em certas aplicações devido a suas propriedades específicas ${ }^{[1]}$.

A poliamida (PA) faz parte de uma família numerosa. De modo geral, todas as poliamidas apresentam boa combinação entre tenacidade e rigidez, com baixo coeficiente de atrito e alta resistência térmica (temperaturas de fusão acima dos $200{ }^{\circ} \mathrm{C}$ e as de deflexão térmica (em carga baixa), além dos $160^{\circ} \mathrm{C}$ ) e química: são inertes a quase todos os tipos de óleos e solventes. Entre as resinas termoplásticas, a PA aceita um maior número de modificações e combinações entre polímeros, permitindo compor uma extensa variedade de formulações, podendo combinar-se com vários outros materiais, aditivos e reforços, possibilitando aplicações diversas ${ }^{[12]}$.

Por sua maior área cristalina, o náilon 6.6 ou PA66 tem, de modo geral, propriedades mecânicas, físicas e químicas mais elevadas. Ainda graças ao elevado grau de cristalinidade, a resina solidifica rapidamente no molde, diminuindo o ciclo de injeção. Além disso, o PA66 absorve menos umidade em relação ao PA6. Considerando $50 \%$ de 
umidade relativa do ar, o PA66 fica estável com teor de 2,5\% de umidade e o PA6, com teor de 3,5\%. Com temperatura de uso contínuo da ordem de $135^{\circ} \mathrm{C}$, o PA66 leva vantagem em aplicações de temperaturas mais elevadas. Seu ponto de fusão é $263^{\circ} \mathrm{C}$, contra $225^{\circ} \mathrm{C}$ do PA6. Desta forma, as propriedades superiores da resina ficam mais evidentes sob altas temperaturas. Já sob temperatura ambiente, o PA6 apresenta maior alongamento e resistência ao impacto superior. Também propicia melhor acabamento superficial do que o 6.6. Sob temperaturas baixas, a PA6 apresenta boa tenacidade e boas propriedades mecânicas, ainda que inferiores às da PA66 ${ }^{[1]}$.

Nos últimos anos, diversas investigações têm sido realizadas sobre o desenvolvimento de compósitos de PA reforçadas com fibras. A adição de fibras como reforço joga um importante papel na resistência, rigidez, estabilidade térmica e propriedades tribológicas do material compósito $^{[13]}$.

Diante deste crescente campo de aplicações fica evidente, portanto, a necessidade de se gerar conhecimento acerca do comportamento dessa categoria de materiais durante operações de usinagem de modo a se obter um desempenho satisfatório durante o corte, além de produzir componentes que atendam aos requisitos estabelecidos. $\mathrm{O}$ fato de o material ser composto por constituintes tão distintos entre si (matriz e fibras) por vezes torna sua usinagem bastante complexa, dificultando a obtenção de superfícies de boa qualidade e de tolerâncias dimensionais e geométricas estreitas ${ }^{[14]}$.

Furação é provavelmente o processo de usinagem convencional de maior importância na fabricação de peças produzidas em compósitos de polímeros reforçados com fibras (CPRF). Isto devido a que, na sua grande maioria, estas peças se apresentam na forma de placas fabricadas por moldagem, conformação ou laminadas, o que requer uma furação para sua posterior montagem. No estudo de projeto de fuselagem de helicópteros realizado por Kassapoglou ${ }^{[15]}$, mostra-se que dentre as diversas atividades necessárias para completar a manufatura de um componente estrutural, operações de furação representam cerca de $8,3 \%$ do custo final dos componentes.

A operação de furação de compósitos tem recebido a atenção de alguns pesquisadores, principalmente devido aos danos causados na entrada e saída da ferramenta, e caracterizados principalmente pela presença de trincas, de laminação da peça ou ainda descolamento das fibras da matriz.

O mecanismo de usinagem de CPRF apresenta características diferentes das presentes na usinagem de metais. Diversos trabalhos têm abordado o efeito dos parâmetros de corte e a geometria na usinagem de CPRF, nas quais se destacam aquelas de matriz epóxi ou poliéster reforçados com fibras de aramida, carbono ou vidro. Entretanto, não existem muitos trabalhos sobre usinagem de $\mathrm{PA}^{[1]}$.

Mata \& Davim ${ }^{[16]}$ apresentaram resultados de um estudo experimental de torneamento de poliamidas com e sem reforço, usando ferramentas de carboneto de tungstênio (metal duro). Os autores afirmam que o material de reforço $(30 \%$ de fibra de vidro) fornece maior tenacidade e resistência, com baixo coeficiente de atrito e de elevada resistência térmica ao material. $\mathrm{O}$ que explicaria o melhor comportamento na rugosidade obtida no torneamento do material com reforço.

Recentemente, Li \& $\mathrm{Xia}^{[13]}$ estudaram o desempenho tribológico de PA6 e PA6 reforçadas com fibras de carbono sobre condições de atrito seco. Os autores concluem que quando se incorpora fibras de carbono, a aderência e a deformação plástica são reduzidas de maneira drástica.

De maneira geral, na usinagem de PA são produzidos cavacos do tipo contínuo. Entretanto, é possível obter cavacos segmentados quando são utilizados avanços grandes e velocidades de corte baixa como mostrado por Gaitonde et al. ${ }^{[14]}$ e que igualmente resultou em melhor acabamento superficial. Da mesma forma, os autores afirmam que a presença das fibras de reforço nos polímeros suportam grande parte da carga aplicada o que reduz diretamente a interação entre o polímero e a ferramenta de corte.

Krishnaraj et al. ${ }^{[3]}$ realizaram um trabalho experimental do tipo fatorial completo sobre furação de placas de polímeros reforçados com fibras de carbono, utilizando brocas de metal duro da classe K20. Neste estudo foram variados os parâmetros de furação de forma a determinar as condições ótimas de usinagem, sendo o diâmetro e circularidade do furo as características avaliadas. Os autores mostram que na medida em que se diminui o avanço, as vibrações regenerativas são reduzidas, obtendo-se furos mais próximos do diâmetro nominal. Isto pode ser atribuído, não só aos parâmetros mais leves, mas também ao maior calor gerado na deformação e pelo atrito.

Tsao \& Hocheng ${ }^{[17]}$ apresentaram um estudo relacionando forces de furação e acabamento superficial obtidos na furação de placas de compósitos poliméricos reforçados com fibras de vidro com brocas de ponta tipo trepanador (candle stick drill). Os resultados experimentais indicam que o diâmetro da broca e o avanço são os fatores mais significativos sobre a força de avanço, enquanto que o avanço e a velocidade de rotação da broca contribuem de maior forma à rugosidade superficial.

Um estudo sobre furação de placas de CPRF/alumínio foi realizado com brocas de metal duro classe K20 por by Zitoune et al. ${ }^{[18]}$. Foram apresentados resultados experimentais que relacionavam velocidade de rotação, avanço e diâmetro da broca sobre a força de avanço. Os resultados mostram que a qualidade dos furos pode ser melhorada por meio da escolha adequada dos parâmetros de corte. Os autores afirmam que maiores tempos de usinagem, contribuem para um aumento da temperatura de usinagem o que provoca um aumento do erro de circularidade. Por outro lado, os efeitos da velocidade de corte sobre o acabamento são menores.

Estudos prévios têm mostrado que a integridade superficial de polímeros de engenharia durante a usinagem é influenciada fortemente pelas propriedades térmicas dos materiais ${ }^{[1]}$. Entretanto, estudos diretos sobre características de usinabilidade e desvios geométricos na furação de polímeros para engenharia ainda não foram apresentados. 
Este estudo tem como objetivo avaliar a usinabilidade de materiais poliméricos com e sem reforço, por meio da análise da pressão específica de corte e dos desvios dimensionais do furo realizado. Os efeitos da variação da velocidade de rotação, do avanço e do ângulo de ponta da broca serão apresentados. Para tanto, placas de material polimérico PA6 e PA66GF30 (reforçado com 30\% de fibra de vidro) obtidas de barras extrudadas serão ensaiadas.

\section{Materiais e Métodos}

Como mostrado anteriormente, as poliamidas PA6 e PA66 apresentam características mecânicas similares, com aplicações semelhantes como engrenagens, mancais, peças automotivas estruturais, entre outros. Desta forma, sua escolha pode estar associada a fatores como disponibilidade ou custos. Entretanto pode-se afirmar que embora a PA6 apresente temperatura de trabalho menor, ele apresenta melhor desempenho quando submetido a longos períodos em temperaturas abaixo da temperatura de fusão (e.g., $150^{\circ} \mathrm{C}$ ). Uma vez, que o processo de usinagem se caracteriza pela grande geração de calor, é possível comparar o desempenho de dois tipos de poliamidas com características de resistência a altas temperaturas diferentes, a saber, PA6 e outra PA66 reforçada com 30\% de fibra de vidro (PA66GF30). Permitindo observar como esta característica de temperatura influencia na usinabilidade e na tolerância final das peças produzidas.

Para a avaliação da usinabilidade das poliamidas, foram realizados ensaios de furação num centro de usinagem com potência no eixo árvore de $7.5 \mathrm{~kW}$ e velocidade de rotação máxima de $8.000 \mathrm{rpm}$. Serão variados os valores de velocidade de rotação (1000; 3000 e 6000 rpm), do avanço $(0,5 ; 0,10$ e $0,15 \mathrm{~mm} / \mathrm{rev})$ e do ângulo de ponta da broca $\left(85^{\circ} ; 115^{\circ}\right.$ e $\left.130^{\circ}\right)$.

As ferramentas utilizadas foram brocas helicoidais de carboneto de tungstênio (classe ISO K20) com diâmetro de $5 \mathrm{~mm}$, duas arestas de corte, ângulo de hélice de $25^{\circ}$ e ângulo de ponta de $85^{\circ}, 115^{\circ}$ e $130^{\circ}$. As três brocas utilizadas têm como código do fabricante (Guhring oHG) as seguintes designações: WN11RN, DIN 6539RN e DIN 8038RN para $130^{\circ}, 115^{\circ}$ e $85^{\circ}$ de ângulo de ponta, respectivamente. A Figura 2 mostra a geometria da ponta das brocas utilizadas.
Os materiais dos corpos de prova utilizados foram: poliamida sem reforço PA6 e poliamida PA66 reforçada com $30 \%$ fibra de vidro, as fibras de vidro estão dispersas na matriz na forma de wiskers com $5 \mathrm{~mm}$ de diâmetro e comprimento médio de $50 \mathrm{~mm}$, denominado comercialmente de PA66GF30. Ambos os materiais comerciais produzidos por extrusão cujas características principais são apresentadas na Tabela 1 .

Os corpos de prova têm diâmetro de $50,8 \mathrm{~mm}$ e espessura média de $10 \mathrm{~mm}$ e foram fixados na máquina através de um suporte de alumínio com furos de $10 \mathrm{~mm}$ de diâmetro na parte inferior para garantir furos passantes sem provocar deflexões consideráveis no corpo de prova. A Figura 3 apresenta os corpos de prova fixados na máquina, durante o processo de usinagem.

$\mathrm{O}$ valor da força de avanço $\left(\mathrm{F}_{F}\right)$ foi obtido por meio de um dinamômetro piezelétrico da marca Kistler modelo 9257BA, o qual apresenta um valor máximo de $500 \mathrm{~N}$ na medição do componente de avanço com repetibilidade de 0,5 N. O dinamômetro está conectado a um amplificador de carga e sistema de aquisição de sinais com freqüência de amostragem de $500 \mathrm{~Hz}$.

Segundo Abrão et al. ${ }^{[19]}$, a relação entre a força de avanço $\left(\mathrm{F}_{F}\right)$ medida durante a furação e a pressão específica de corte $\left(k_{\mathrm{f}}\right)$, pode ser obtida por meio da Equação 1:

$$
k_{f}=\frac{2 \cdot F_{f}}{f \cdot d}
$$

onde $F_{\mathrm{f}}$ corresponde a força de avanço $(\mathrm{N}), f$ o avanço $(\mathrm{mm} / \mathrm{rev})$, e $d$ o diâmetro da broca $(\mathrm{mm})$.

$\mathrm{O}$ valor do diâmetro máximo $\left(\mathrm{D}_{\max }\right)$ do furo foi medido por meio de uma máquina de medir por coordenadas (MMC) Tesa Micro-hite 3D com precisão de $1 \mathrm{~mm}$. Para obtenção dos desvios de circularidade a MMC utiliza 7 pontos para definir e calcular o círculo. A Figura 4 mostra o equipamento utilizado para medição da circularidade dos furos usinados

Os efeitos da variação da velocidade de rotação, do avanço e do ângulo de ponta da broca sobre a pressão específica de corte $\left(\mathrm{k}_{\mathrm{s}}\right)$ e sobre o desvio do valor nominal do diâmetro $\left(\mathrm{D}_{\max }\right)$ serão apresentados por meio de gráficos.

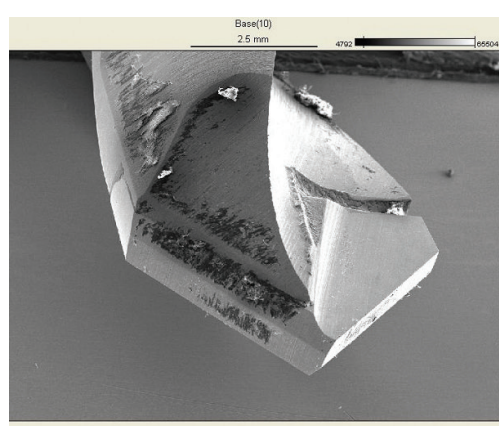

DIN 8038RN - 85०

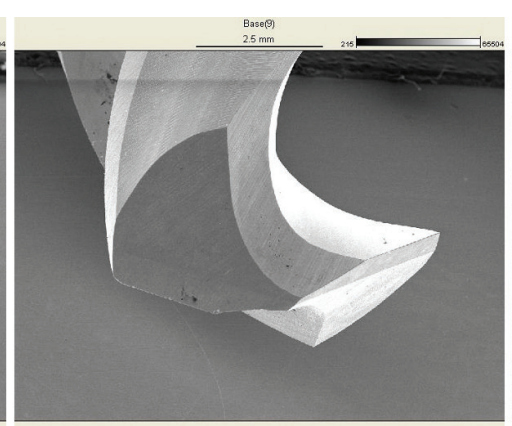

DIN6539RN - 115

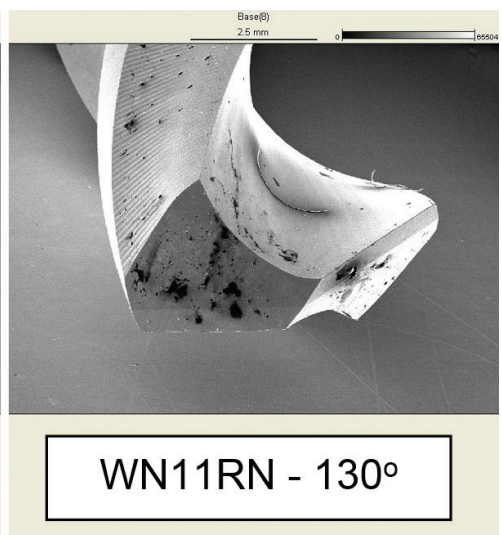

Figura 2. Detalhe da ponta das brocas utilizadas. 
Tabela 1. Composição e propriedades da PA6 e do compósito PA66GF30.

\begin{tabular}{|c|c|c|c|}
\hline \multirow{2}{*}{ Propriedades } & \multicolumn{2}{|c|}{ Material } & \multirow{2}{*}{ Unidades } \\
\hline & PA6 & PA66GF30 & \\
\hline Fibras de Reforço & ------ & $\begin{array}{l}\text { wiskers de fibra de vidro }(\varnothing 5 \\
\mu \mathrm{m} \times 50 \mu \mathrm{m} \text { de compr.) }\end{array}$ & -- \\
\hline Modulo de elasticidade & 1400 & 3200 & $\mathrm{MPa}$ \\
\hline Dureza Rockwell & M85 & M76 & - \\
\hline Resistência ao Impacto Charpy & Sem Fratura & 50 & $\mathrm{KJ} / \mathrm{m}^{2}$ \\
\hline Resistência à tração & 76 & 100 & $\mathrm{MPa}$ \\
\hline Temperatura de fusão & 220 & 255 & ${ }^{\circ} \mathrm{C}$ \\
\hline Densidade & 1.14 & 1.29 & $\mathrm{~g} / \mathrm{cm}^{3}$ \\
\hline Coeficiente de expansão térmica $\left(<150^{\circ} \mathrm{C}\right)$ & $90 \times 10^{6}$ & $50 \times 10^{6}$ & $\mathrm{~m}(\mathrm{~m} \cdot \mathrm{k})$ \\
\hline Coeficiente de expansão térmica $\left(>150^{\circ} \mathrm{C}\right)$ & $105 \times 10^{6}$ & $60 \times 10^{6}$ & $\mathrm{~m}(\mathrm{~m} \cdot \mathrm{k})$ \\
\hline
\end{tabular}

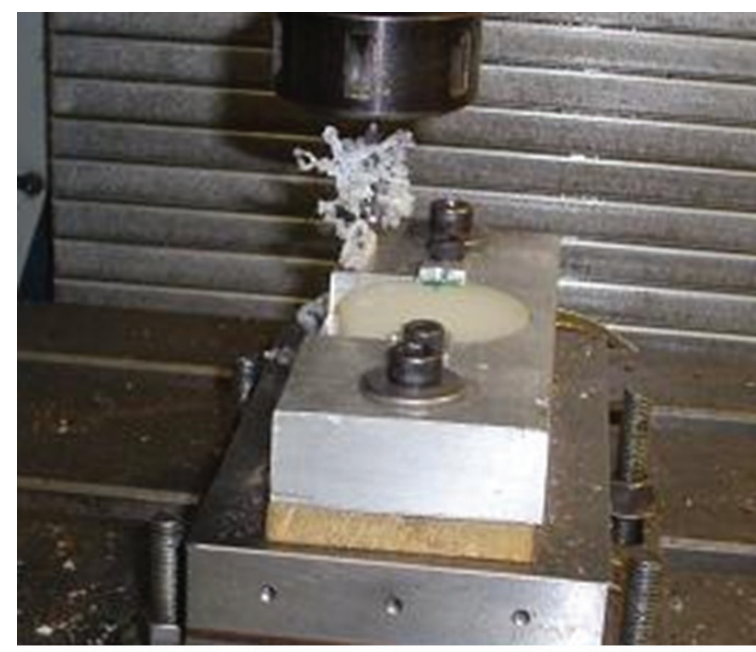

(a)

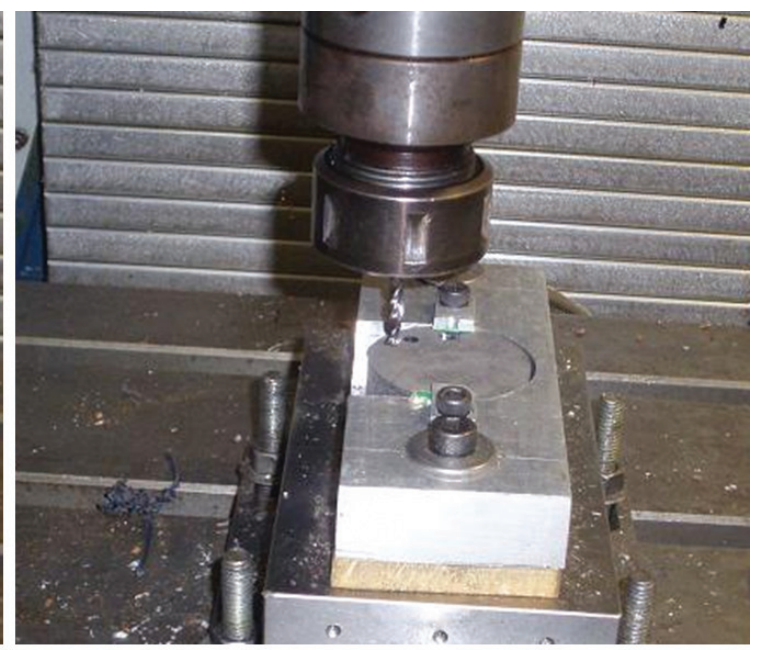

(b)

Figura 3. Sistema de fixação dos corpos de prova de poliamida no processo de furação: a) PA6 e b) PA66GF30.

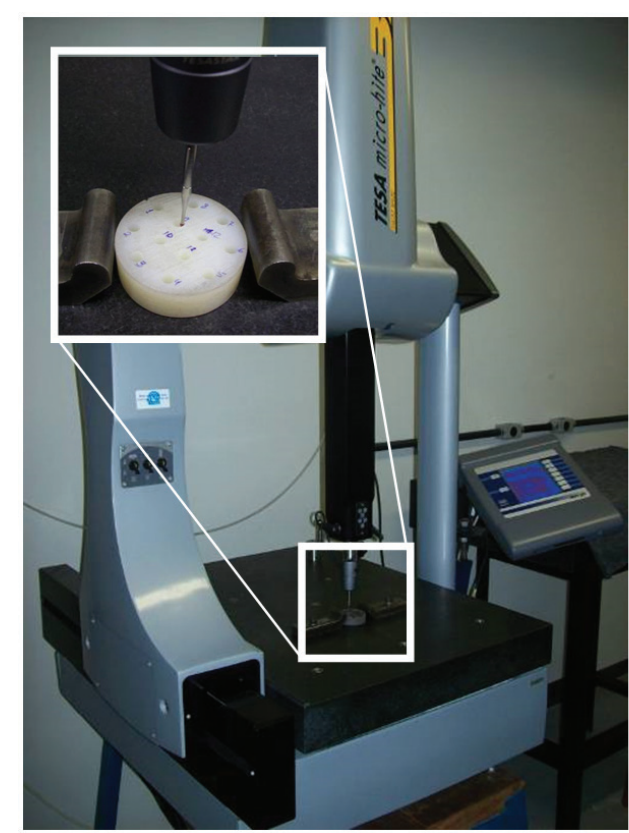

(a)

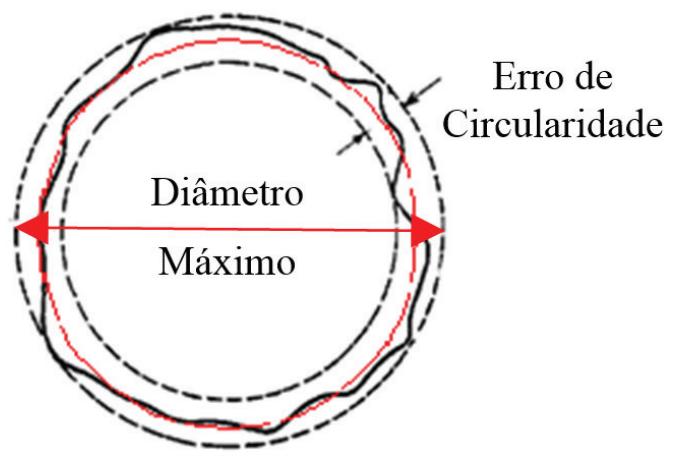

(b)

Figura 4. Medição de circularidade: a) Maquina de medir por coordenadas Tesa Micro-hite 3D; b) diâmetro máximo do furo. 


\section{Resultados}

A Tabela 2 mostra os parâmetros de corte empregados no ensaio, assim como os resultados de pressão específica de corte $\left(\mathrm{k}_{\mathrm{f}}\right)$ e diâmetro máximo do furo $\left(\mathrm{D}_{\max }\right)$, obtidos para três velocidades de corte, três avanços e três brocas diferentes, onde cada teste foi realizado duas vezes.
A Figura 5 mostra a resposta da pressão específica de corte para variação da velocidade de rotação e do avanço. Nota-se que a pressão específica de corte $\left(\mathrm{k}_{\mathrm{f}}\right)$ para o material sem reforço (PA6) é muito superior à obtida na furação do material reforçado com wiskers de fibra de vidro (PA66GF30). Esta característica pode ser atribuída ao maior efeito da temperatura sobre a PA6 cuja temperatura

Tabela 2. Resultados obtidos para a variação de parâmetros na furação de PA6 e PA66GF30.

\begin{tabular}{|c|c|c|c|c|c|c|c|}
\hline \multicolumn{8}{|c|}{ Poliamida PA6 } \\
\hline \multirow[b]{2}{*}{$\begin{array}{c}\text { Velocidade } \\
\text { (rpm) }\end{array}$} & \multirow[b]{2}{*}{$\begin{array}{c}\text { Avanço } \\
(\mathrm{mm} / \mathrm{rev})\end{array}$} & \multicolumn{2}{|c|}{ Broca $85^{\circ}$} & \multicolumn{2}{|c|}{ Broca $115^{\circ}$} & \multicolumn{2}{|c|}{ Broca $135^{\circ}$} \\
\hline & & $\begin{array}{c}\mathbf{k}_{\mathrm{f}} \\
\left(\mathrm{N} / \mathbf{m m}^{2}\right) \\
\end{array}$ & $\begin{array}{r}D_{\text {máx }} \\
(\mathbf{m m})\end{array}$ & $\begin{array}{c}\mathbf{k}_{\mathrm{f}} \\
\left(\mathrm{N} / \mathbf{m m}^{2}\right)\end{array}$ & $\begin{array}{c}D_{\text {máx }} \\
(\mathbf{m m})\end{array}$ & $\begin{array}{c}\mathbf{k}_{\mathrm{f}} \\
\left(\mathrm{N} / \mathbf{m m}^{2}\right)\end{array}$ & $\begin{array}{c}D_{\text {máx }} \\
(\mathbf{m m})\end{array}$ \\
\hline \multirow[t]{3}{*}{1500} & 0,05 & 871,44 & 5,035 & 369,36 & 4,951 & 529,28 & 5,012 \\
\hline & 0,10 & 470,86 & 4,99 & 185,56 & 4,975 & 302,62 & 5,059 \\
\hline & 0,15 & 319,51 & 4,981 & 123,77 & 4,987 & 241,21 & 5,045 \\
\hline \multirow[t]{3}{*}{3000} & 0,05 & 670,56 & 5,053 & 310,20 & 5,017 & 475,08 & 5,039 \\
\hline & 0,10 & 374,20 & 5,04 & 150,86 & 4,973 & 283,62 & 5,033 \\
\hline & 0,15 & 273,47 & 4,989 & 122,44 & 4,968 & 218,09 & 5,031 \\
\hline \multirow[t]{3}{*}{6000} & 0,05 & 458,64 & 5,085 & 254,60 & 5,107 & 435,48 & 5,061 \\
\hline & 0,10 & 259,06 & 5,172 & 133,74 & 4,991 & 264,44 & 5,075 \\
\hline & 0,15 & 190,45 & 5,205 & 100,87 & 4,979 & 200,40 & 5,052 \\
\hline \multicolumn{8}{|c|}{ Poliamida PA66GF30 } \\
\hline & & \multicolumn{2}{|c|}{ Broca $85^{\circ}$} & \multicolumn{2}{|c|}{ Broca $115^{\circ}$} & \multicolumn{2}{|c|}{ Broca $135^{\circ}$} \\
\hline $\begin{array}{l}\text { Velocidade } \\
\text { (rpm) }\end{array}$ & $\begin{array}{c}\text { Avanço } \\
(\mathrm{mm} / \mathrm{rev})\end{array}$ & $\begin{array}{c}\mathbf{k}_{\mathrm{f}} \\
\left(\mathrm{N} / \mathbf{m m}^{2}\right)\end{array}$ & $\begin{array}{c}D_{\text {máx }} \\
(\mathrm{mm})\end{array}$ & $\begin{array}{c}\mathbf{k}_{\mathrm{f}} \\
\left(\mathrm{N} / \mathbf{m m}^{2}\right)\end{array}$ & $\begin{array}{c}D_{\text {máx }} \\
(\mathbf{m m})\end{array}$ & $\begin{array}{c}\mathbf{k}_{\mathrm{f}} \\
\left(\mathrm{N} / \mathbf{m m}^{2}\right)\end{array}$ & $\begin{array}{c}D_{\text {máx }} \\
(\mathrm{mm})\end{array}$ \\
\hline \multirow[t]{3}{*}{1500} & 0,05 & 185,40 & 5,015 & 158,48 & 5,001 & 233,40 & 5,079 \\
\hline & 0,10 & 118,34 & 5,000 & 91,08 & 5,002 & 153,88 & 5,086 \\
\hline & 0,15 & 99,59 & 4,996 & 69,77 & 5,002 & 129,41 & 5,091 \\
\hline \multirow[t]{3}{*}{3000} & 0,05 & 166,36 & 4,998 & 144,24 & 5,003 & 192,08 & 5,089 \\
\hline & 0,10 & 105,38 & 5,000 & 83,58 & 5,005 & 147,96 & 5,088 \\
\hline & 0,15 & 83,65 & 5,006 & 62,78 & 5,006 & 124,17 & 5,071 \\
\hline \multirow[t]{3}{*}{6000} & 0,05 & 158,92 & 5,003 & 129,20 & 5,018 & 191,68 & 5,131 \\
\hline & 0,10 & 98,80 & 5,004 & 77,74 & 5,006 & 143,32 & 5,111 \\
\hline & 0,15 & 78,41 & 5,006 & 57,65 & 5,017 & 119,91 & 5,094 \\
\hline
\end{tabular}

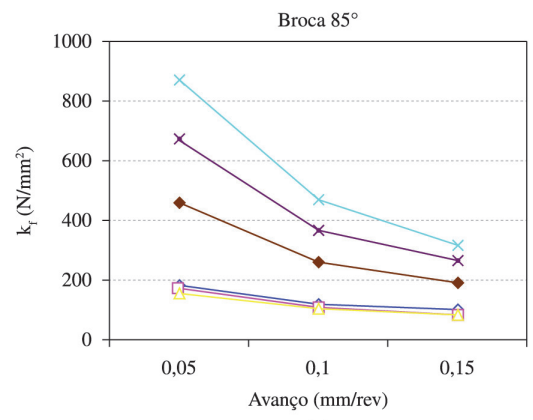

(a)

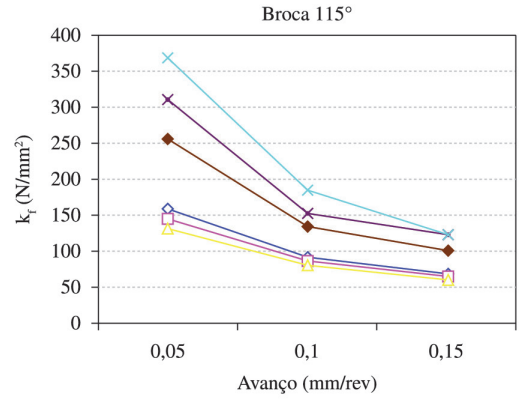

(b)
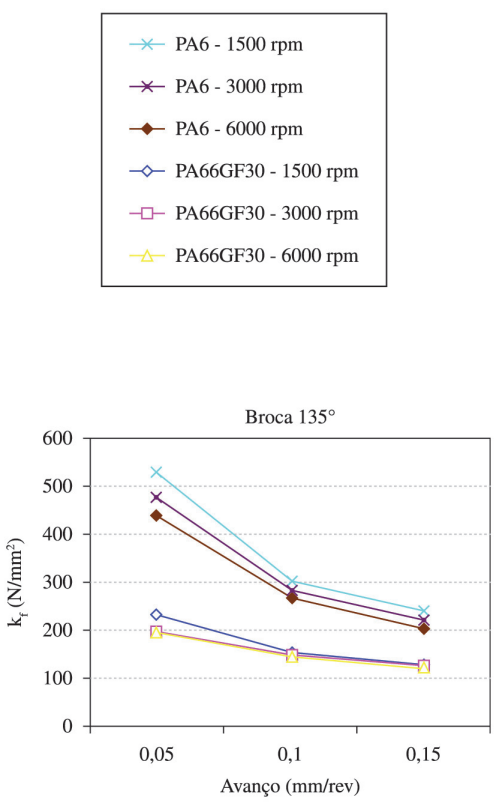

(c)

Figura 5. Resultado da pressão específica de corte $\left(\mathrm{k}_{\mathrm{f}}\right)$ para furação de PA6 e PA66GF30: a) Broca de $85^{\circ}$; b) Broca de $115^{\circ}$; c) Broca de $135^{\circ}$. 


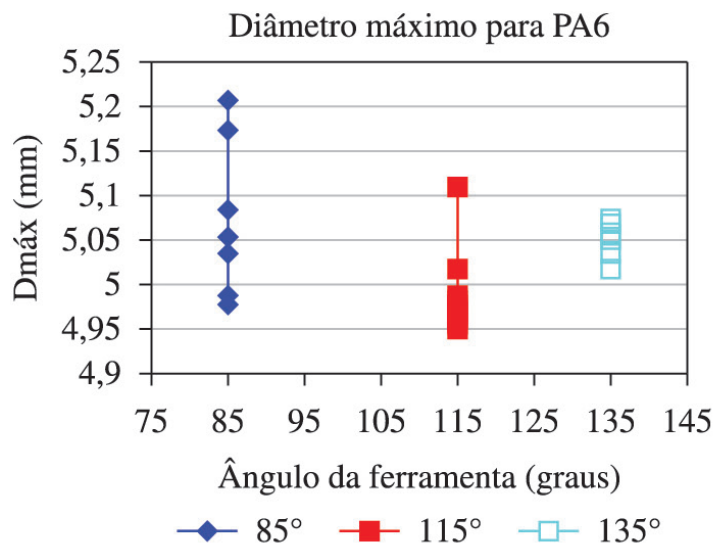

(a)

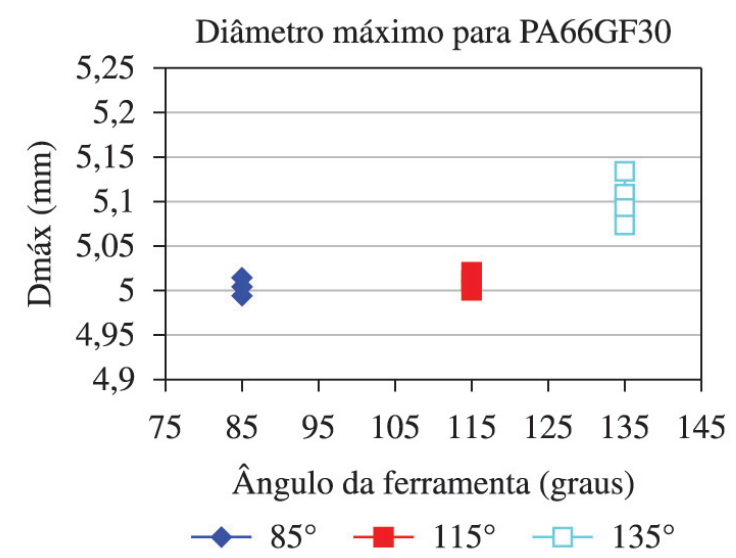

(b)

Figura 6. Desvio máximo obtidos na furação com brocas de ângulo de ponta diferente para os dois materiais: a) PA6 e b) PA66GF30.

de fusão mais baixa associada à menor resistência, provoca a produção de cavacos maiores (emaranhados), como pode ser observado na Figura 3a). A dificuldade de expulsão dos cavacos da região de corte e a maior aderência do PA6 na ferramenta implicam em um aumento substancial das forças de usinagem.

Com o objetivo de modelar o processo de usinagem, diversos pesquisadores têm desenvolvido equações para determinar a pressão específica de corte e sua variação com a alteração dos parâmetros de corte ${ }^{[20]}$. Neste sentido, e devido à menor variabilidade de $\mathrm{k}_{\mathrm{f}}$, podem-se obter modelos mais simples e com maior precisão na determinação da força teórica de usinagem para PA66GF30.

A Figura 6 mostra a variação do Diâmetro Máximo $\left(\mathrm{D}_{\text {máx }}\right)$ resultante da ação das brocas com ângulo de ponta diferentes para a Poliamida sem reforço (PA6) e a Poliamida reforçada com fibra de vidro (PA66GF30). Nota-se que os desvios obtidos na usinagem de material sem reforço apresentam maior desvio, o que pode ser explicado devido à menor temperatura de transição vítrea deste material, que a torna mais susceptível ao calor gerado no processo.

Dentro deste mesmo raciocínio, as brocas com ângulos de ponta mais agudos, apresentam uma maior área de contato, o que provoca uma área de aquecimento, o que fica mais evidente no aumento do $\mathrm{D}_{\max }$ no material sem reforço (Figura 6a). Isto pode ser atribuído à sua menor temperatura de fusão.

Como pode se observar na Figura 6b, o comportamento da poliamida reforçada (PA66GF30) durante a usinagem se assemelha ao da maioria dos materiais metálicos, onde o menor desvio do valor nominal do diâmetro do furo $\left(\mathrm{D}_{\max }\right)$ esta associado, em maior grau, ao menor nível de esforço e o conseqüente menor nível de vibrações obtidas em ângulos de ponta da broca menores (pontas mais agudas).

\section{Conclusões}

Este trabalho apresentou um estudo comparativo do comportamento da pressão específica de corte $\left(\mathrm{k}_{\mathrm{f}}\right)$ e dos desvios de circularidade obtidos na furação de poliamida reforçada com fibra de vidro (PA66 GF30) e poliamida sem reforço (PA6).

O estudo permitiu constatar que os níveis de força para a furação de poliamidas sem reforço (PA6) é significativamente maior que na usinagem de poliamidas reforçadas (PA66GF30). Isto pode ser atribuído ao maior efeito da temperatura sobre a PA6 cuja temperatura de fusão mais baixa e que associada à menor resistência, apresenta uma produção de cavacos mais longos (emaranhados). Desta forma, a maior dificuldade de expulsão dos cavacos da região de corte e a maior aderência do PA6 na ferramenta implicam em um aumento substancial das forças de usinagem.

Os resultados de pressão específica de corte $\left(\mathrm{k}_{\mathrm{f}}\right)$ obtida a partir da força de avanço na furação de materiais poliméricos diminuem com o aumento dos parâmetros de corte (velocidade de corte e avanço).

Da mesma forma pode-se concluir que ferramentas com menor ângulo de ponta apresentam em geral melhor desempenho na furação de PA66GF30 nestas condições de corte. Entretanto, nota-se que os desvios $\mathrm{D}_{\text {máx }}$ obtidos na usinagem de material sem reforço PA66 apresentam maior desvio, o que pode ser explicado devido à menor temperatura de transição vítrea deste material, que a torna mais susceptível ao calor gerado no processo.

Finalmente, o comportamento do PA66GF30 durante a usinagem se assemelha ao da maioria dos materiais metálicos, onde o menor desvio do valor nominal do diâmetro do furo $\left(\mathrm{D}_{\max }\right)$ está associado, em maior grau, ao menor nível de esforço e o conseqüente menor nível de vibrações obtidas em ângulos de ponta da broca menores (pontas mais agudas).

\section{Referências Bibliográficas}

1. Campos Rubio, J. C.; Silva, L. J.; Leite, W. O.; Panzera, T. H.; Ribeiro Filho, S. L. M. \& Davim, J. P. - Compos., Part B Eng., 55, p.338 (2013). http://dx.doi.org/10.1016/j. compositesb.2013.06.042.

2. Vaidya, U. - "Composites for automotive, truck and mass transit”, DEStech Publications, Inc., Lancaster (2011). 
3. Krishnaraj, V.; Prabukarthi, A.; Ramanathan, A.; Elanghovan, N.; Senthil Kumar, M.; Zitoune, R. \& Davim, J. P. - Compos., Part B Eng., 43, p.1791 (2012). http://dx.doi.org/10.1016/j. compositesb.2012.01.007.

4. Daniel, I. M. \& Ishai, O. - "Engineering mechanics of composite materials", Oxford University Press, New York (1994).

5. Ghassemieh, E. - "Materials in automotive application, state of the art and prospects", in: New trends and developments in automotive industry, cap.20, Marcello Chiaberge (ed.), InTech Publisher (2011). http://dx.doi.org/10.5772/13286.

6. Babjak, S.; Dúbravčík, M. \& Kender, S. - Annals of F.E.H. Int. J. of Eng., 10, p.77 (2012).

7. Carpenter, J. A. - "Challenges and opportunities for automotive composites", in: Proceedings of SPE Automotive Composites Conference and Exposition, CD-ROM, p.1, Troy - MI, 16-18 set (2008).

8. Gabriel, A. P.; Grochau, I. H.; Santana, R. M. C.; Veit, H. M. - Polímeros, 23, p.823 (2013). http://dx.doi.org/10.4322/ polimeros.2014.004.

9. Engine Technology Industrial - ETI. (2012). Disponível em: $<$ http://www.enginetechnologyinternational.com $>$. Acesso em: 13 fev. 2013ano.

10. American Motorcyclist Association - AMA. (2012) Disponível em: $<$ http://www.amaproracing.com $>$. Acesso em: 25 fev. 2014.

11. McCrum, N. G. \& Buckley, C. P. - "Principles of polymer engineering”, Oxford University Press, New York (1997).
12. Fantoni, R. F. - Polímeros., 22, p.1 (2012). http://dx.doi. org/10.1590/S0104-14282012000100003.

13. Li, J. \& Xia, Y. C. - J. Thermoplast Compos. Mater., 23, p.337 (2010). http://dx.doi.org/10.1177/0892705708103397.

14. Gaitonde, V. N.; Karnik, S. R.; Campos Rubio, J.; Correia, A. E. \& Davim, J. P. - J. Compos. Mater., 46, p.2659 (2012). http://dx.doi.org/10.1177/0021998311431640.

15. Kassapoglou, C. - Compos. Part A., 30, p.895 (1999). http:// dx.doi.org/10.1016/S1359-835X(98)00191-2.

16. Mata, F. \& Davim, J. P. - Int. J. Mat. Prod. Technol., 32, p.295 (2008). http://dx.doi.org/10.1504/IJMPT.2008.018987.

17. Tsao, C. C. \& Hocheng, H. - J. Mater. Process. Technol., 203, p.342 (2008). http://dx.doi.org/10.1016/j. jmatprotec.2006.04.126.

18. Zitoune, R.; Krishnaraj, V.; Sofiane Almabouacif, B.; Collombet, F.; Sima, M. \& Jolin, A. - Compos., Part B Eng., 43, p.1480 (2012). http://dx.doi.org/10.1016/j. compositesb.2011.08.054.

19. Abrão, A. M.; Faria, P. E.; Rubio, J. C.; Reis, P. \& Davim, J. P. - J. Mat. Proc. Tech., 186, p.1 (2007). http://dx.doi. org/10.1016/j.jmatprotec.2006.11.146.

20. Trent, E. M. \& Wright, P. K. - "Metal cutting", 4. ed., Butterworth-Heinemann, Boston (2000).

Enviado: Out. 24, 2013

Reenviado: Abr. 23, 2014 Aceito: Maio 19, 2014 